\title{
Near-Capacity Three-Stage Downlink Iteratively Decoded Generalized Layered Space-Time Coding with Low Complexity
}

\author{
Lingkun Kong, Soon Xin Ng, and Lajos Hanzo \\ School of ECS, University of Southampton, SO17 1BJ, United Kingdom. \\ Tel: +44-23-8059 3125, Fax: +44-23-8059 4508 \\ Email: $\{1 \mathrm{k} 06 \mathrm{r}, \mathrm{sxn}, \mathrm{lh}\} @$ @ecs.soton.ac.uk, http://www-mobile.ecs.soton.ac.uk
}

\begin{abstract}
This paper presents a low complexity iteratively detected space-time transmission architecture based on Generalized Layered Space-Time (GLST) codes and IRregular Convolutional Codes (IRCCs). The GLST combines the benefits of the Vertical Bell-labs LAyered Space-Time (V-BLAST) scheme and SpaceTime Coding (STC). The GLST is serially concatenated with a Unity-Rate Code (URC) and an IRCC which are used to facilitate near-capacity operation with the aid of an EXtrinsic Information Transfer (EXIT) chart based design. Reducedcomplexity iterative Successive Interference Cancellation (SIC) is employed in the GLST decoder, instead of the significantly more complex Maximum Likelihood (ML) detection. For the sake of approaching the maximum achievable rate, iterative decoding is invoked to achieve decoding convergence by exchanging extrinsic information across the three serial component decoders. Finally, it is shown that the SIC-based iteratively detected IRCC-URCGLST system is capable of providing a trade-off between the affordable computational complexity and the system throughput.
\end{abstract}

\section{INTRODUCTION}

Recent information theoretic studies have shown that the capacity of a Multiple-Input Multiple-Output (MIMO) system [1]-[4] is significantly higher than that of a SingleInput Single-Output (SISO) system. MIMO techniques are also capable of achieving both multiplexing gain and diversity gain. In [5], Wolniansky et al. proposed the popular multilayer MIMO structure, referred to as the Vertical Bell-labs LAyered Space-Time (V-BLAST) scheme, which is capable of increasing the throughput without any increase in the transmitted power or the system's bandwidth. Although it was primarily designed for exploiting the transmit multiplexing gain, it is worth noting that upon increasing the number of antennas, typically the achievable transmit diversity gain also increases at the cost of an increased receiver complexity.

In contrast to spatial multiplexing techniques, Alamouti [6] discovered a transmit diversity scheme, referred to as a Space-Time Block Code (STBC), where the prime concern is achieving diversity gain. The attractive benefits of Alamouti's design motivated Tarokh et al. [7] to generalize Alamouti's scheme to an arbitrary number of transmit antennas. Another transmit diversity scheme, referred to as Space-Time Trellis Coding (STTC) was invented by Tarokh et al. in [8], which is capable of achieving both spatial diversity gain and coding

The financial support of the China Scholarship Council, as well as that of the EPSRC UK, the EU under the auspices of the Optimix project is gratefully acknowledged. gain or time diversity gain. However, these conventional STBC and STTC schemes achieve at most the same data rate as an uncoded single-antenna system. Hence, a MIMO scheme attaining both multiplexing gain and diversity gain is attractive. Various hybrid BLAST and STTC schemes have been proposed in [9], [10]. The Generalized Layered SpaceTime (GLST) code [10] is constructed as a composite of the V-BLAST scheme and Space-Time Coding (STC), which strikes a trade-off between transmission efficiency and error probability. In [10], iterative Successive Interference Cancellation (SIC) was proposed to achieve full receive diversity, which made it possible to achieve the same diversity order as classic Maximum Likelihood (ML) detection at a fraction of its complexity.

For the sake of decoding convergence to an infinitesimally low bit error ratio (BER), the GLST scheme is serially concatenated with outer codes for iteratively exchanging mutual information between the constituent decoders. The decoding convergence of iteratively decoded schemes can be analysed using EXtrinsic Information Transfer (EXIT) charts [11], [12]. Tüchler and Hagenauer [12], [13] proposed the employment of IRregular Convolutional Codes (IRCCs) in serial concatenated schemes, which are constituted by a family of convolutional codes having different rates, in order to design a near-capacity system. They were specifically designed with the aid of EXIT charts to improve the convergence behaviour of iteratively decoded systems. As a further advance, it was shown in [14], [15] that a recursive Unity-Rate Code (URC) should be employed as an intermediate code in order to improve the attainable decoding convergence. The novel contribution of this treatise is that we use EXIT charts to design an iteratively decoded nearcapacity three-stage IRCC-URC-GLST scheme. Specifically, the computational complexity of this concatenated system is substantially reduced at the cost of a modest reduction in the maximum achievable rate compared to ML detection, owing to the employment of the low-complexity but suboptimum SIC in the GLST decoder.

The rest of this paper is organised as follows. In Section II, a brief description of the serially concatenated and iteratively decoded scheme is presented. Section III specifies the encoding and decoding processes designed for the GLST system. The EXIT chart aided iterative decoder design is detailed in Section IV, while our simulation results and discussions are provided in Section V. Finally, we conclude in Section VI. 


\section{System OVERVIEW}
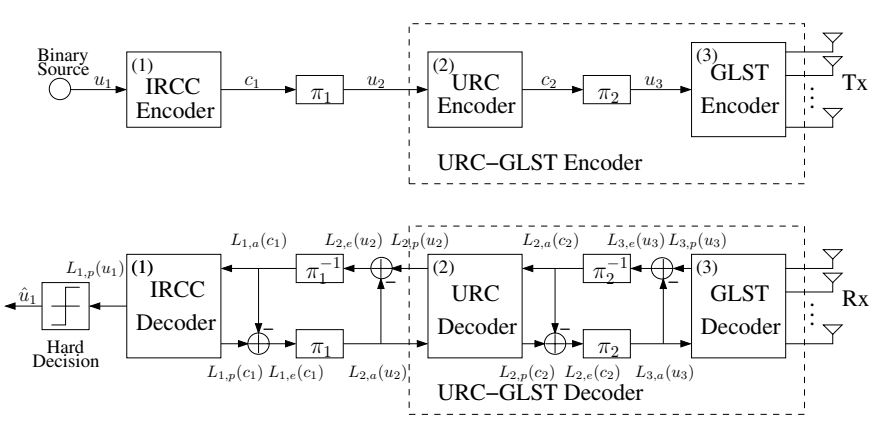

Fig. 1. Schematic of the proposed IRCC-URC-GLST scheme.

The schematic of the proposed serially concatenated system is illustrated in Fig. 1. At the transmitter side, IRCCs [12], [13] are employed for encoding specifically optimized fractions of the input stream, where each fraction's code rate was designed for achieving a near-capacity performance with the aid of EXIT charts [11]. Again, a recursive URC was amalgamated with the above-mentioned GLST in Fig. 1 as the inner code for assisting the non-recursive GLST scheme in achieving decoding convergence to an infinitesimally low BER at near-capacity Signal-to-Noise Ratios (SNRs). The GLST encoder partitions the long bit stream emanating from the intermediate URC encoder into several substreams and each substream is spacetime encoded separately, as will be detailed in Section III. Two different high-length bit interleavers are introduced between the three component encoders so that the input bits of the URC and GLST encoders can be rendered independent of each other, which guarantees that the assumptions facilitating the application of EXIT charts are complied with [11].

At the receiver side, according to Fig. 1, an iterative decoding procedure is operated, which employs three A Posteriori Probability (APP)-based decoders. The received signals are first decoded by the APP-based GLST decoder in order to produce the a priori Log-Likelihood Ratio (LLR) values $L_{2, a}\left(c_{2}\right)$ of the coded bits $c_{2}$. The URC decoder processes the information forwarded by the GLST decoder in conjunction with the a priori LLR values $L_{2, a}\left(u_{2}\right)$ of the information bits $u_{2}$ in order to generate the a posteriori LLR values $L_{2, p}\left(u_{2}\right)$ and $L_{2, p}\left(c_{2}\right)$ of the information bits $u_{2}$ and the coded bits $c_{2}$, respectively. In the scenario when iterations are needed within the amalgamated "URC-GLST" decoder so as to achieve a near-capacity performance, the a priori LLRs $L_{2, a}\left(c_{2}\right)$ are subtracted from the a posteriori LLR values $L_{2, p}\left(c_{2}\right)$ and then they are fed back to the GLST decoder as the a priori information $L_{2, a}\left(u_{3}\right)$ through the interleaver $\pi_{2}$. Similarly, the a priori LLR values of the URC decoder are subtracted from the a posteriori LLR values produced by the Maximum Aposteriori Probability (MAP) algorithm [16], for the sake of generating the extrinsic LLR values $L_{2, e}\left(u_{2}\right)$. Next, the soft bits $L_{1, a}\left(c_{1}\right)$ are passed to the IRCC decoder in order to compute the a posteriori LLR values $L_{1, p}\left(c_{1}\right)$ of the IRCC encoded bits $c_{1}$. During the last iteration, only the LLR values $L_{1, p}\left(u_{1}\right)$ of the original information bits $u_{1}$ are required, which are passed to the hard-decision block in order to estimate the source bits. As seen in Fig. 1, the extrinsic information $L_{1, e}\left(c_{1}\right)$ is generated by subtracting the a priori information from the a posteriori information, which is fed back to the URC decoder as the a priori information $L_{2, a}\left(u_{2}\right)$ through the interleaver $\pi_{1}$.

\section{Generalized Layered Space-Time Code}

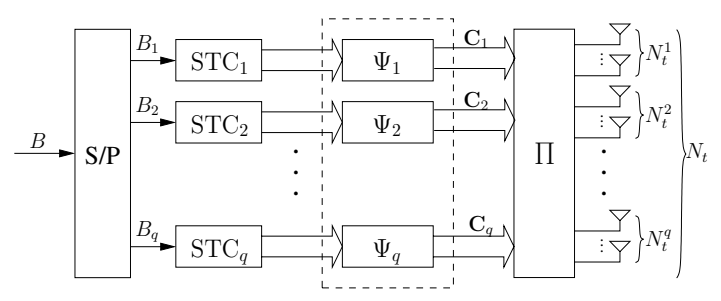

Fig. 2. GLST schematic using the temporal interleaver $\Psi_{j}$ and spatial interleaver $\Pi$.

The GLST scheme of Fig. 2 and Fig. 3 is specified as follows. At the transmitter side of Fig. 2, the antennas are divided into several groups and each group utilizes an STC encoder. At the receiver side of Fig. 3, we avoid the potentially excessive complexity of jointly detecting all groups, which may be achieved in the spirit of the V-BLAST detection algorithm [5], [17] using the reduced-complexity SIC based detection scheme of [10], where the space-time code of each individual group was processed successively, as detailed later in Section III-B. We also present a significantly complex iterative ML detection scheme in Fig. 4 for comparison. Therefore, it is clearly seen that the GLST scheme can be viewed as a beneficial amalgam of a V-BLAST and a STC scheme. With the advent of the STC employed, we will show that this GLST architecture is capable of achieving a higher spatial diversity compared to the conventional V-BLAST scheme. As an added benefit, the overall transmission system's throughput is significantly higher than that of the STC scheme owing to the BLAST-like layered architecture.

\section{A. Encoding}

Consider now a point-to-point wireless communication link equipped with $N_{t}$ transmit and $N_{r}$ receive antennas, where the signal to be transmitted over the $j$ th antenna at time instant $k$ is denoted by $c_{k}^{j}$. In this contribution, we consider transmissions over a temporally uncorrelated flat Rayleigh fading channel. Therefore, the signal received by each antenna is constituted by the superposition of independently Rayleigh faded transmitted signals. The signal received by the $i$ th antenna at time instant $k$ is given by

$$
r_{k}^{i}=\sum_{j=1}^{N_{t}} h_{i, j} c_{k}^{j}+v_{k}^{i},
$$

where $h_{i, j}$ is the complex-valued channel coefficient between the $j$ th transmit and the $i$ th receive antenna, which is modeled by the samples of independent complex Gaussian random variables having a zero mean and a variance of 0.5 per dimension. Furthermore, $v_{k}^{i}$ is the Additive White Gaussian Noise (AWGN) encountered by the $i$ th antenna at time instant 


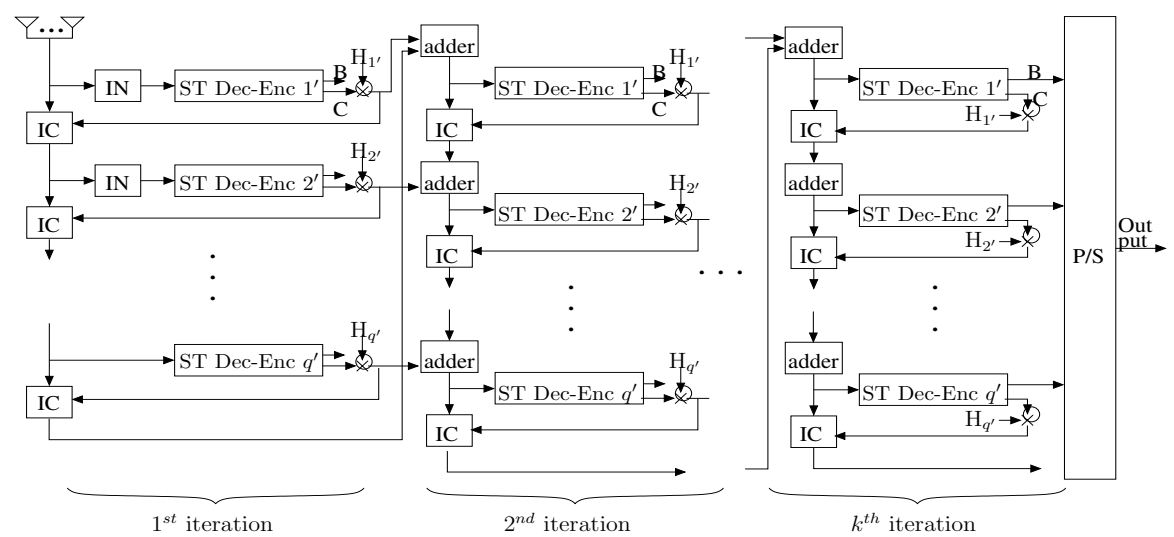

Fig. 3. Iterative SIC based detection of the GLST scheme, where "IN" and "IC" denote the group interference nulling module and the group interference cancellation module respectively, while "ST Dec-Enc $j^{\prime}$ " represents the space-time decoding and re-encoding module.

$k$ and modeled by the samples of independent complex Gaussian random variables having a zero mean and a variance of $N_{0} / 2$ per dimension.

The GLST encoding process is illustrated in Fig. 2. We assume that a block of $B$ information bits is input to a serialto-parallel $(\mathrm{S} / \mathrm{P})$ converter, which partitions this bit stream into $q$ groups, which we refer to as layers having lengths of $B_{1}, B_{2}, \ldots, B_{q}$, where we have $B_{1}+B_{2}+, \ldots,+B_{q}=B$ bits in total. Then, each group of $B_{j}$ bits, for $1 \leq j \leq q$, is separately encoded in Fig. 2 by a component encoder $S T C_{j}$ associated with $N_{t}^{j}$ number of transmit antennas, where we have $N_{t}^{1}+N_{t}^{2}+, \ldots,+N_{t}^{q}=N_{t}$. The resultant $\left(N_{t}^{j} \times K\right)$ element codeword matrix $\mathbf{C}_{j}$ of $S T C_{j}$ will be transmitted by the $N_{t}^{j}$ transmit antennas of Fig. 2 during $K$ symbol intervals. We refer to the $k$ th column $\mathbf{c}_{j, k}$ of $\mathbf{C}_{j}$ as the symbol vector generated by group $j$ at time instant $k$. Following space-time encoding, the symbol vectors of each group are passed through an independent vector-based temporal interleaver $\Psi_{j}$ followed by the common spatial interleaver $\Pi$ of Fig. 2 . The vectorbased temporal interleavers $\Psi_{j}$ represented by the dashed block of Fig. 2 are used for the codewords generated by the different groups, for the sake of eliminating the effects of bursty error propagation among different groups during the decoding iterations [10]. Furthermore, the spatial interleaver $\Pi$ of Fig. 2 may be considered to be a mapping of the spacetime encoded symbol vectors to a certain group of transmit antennas, which provides additional spatial diversity. We can express the received baseband data in matrix form as:

$$
\mathbf{r}_{k}=\mathbf{H}_{1, k} \mathbf{c}_{1, k}+\mathbf{H}_{2, k} \mathbf{c}_{2, k}+\cdots+\mathbf{H}_{q, k} \mathbf{c}_{q, k}+\mathbf{v}_{k},
$$

where $\mathbf{H}_{j, k}$ denotes the $\left(N_{r} \times N_{t}^{j}\right)$-element subchannel matrix of group $j$ at time instant $k$.

\section{B. Iterative SIC Detection}

Again, for the sake of maintaining an affordable computational complexity, a reduced-complexity SIC based detection scheme was proposed in [10] instead of ML detection. For the sake of low complexity, the signals are detected layer-bylayer instead of an ML-style joint-detection and hence this BLAST-like structure fails to achieve full receive diversity, because the earlier a layer is detected, the lower its diversity order. In order to maximize the attainable receive diversity gain, the SIC-based iterative decoding scheme of [10] was invoked, which is depicted in Fig. 3.

In the same spirit as proposed in the V-BLAST scheme [18], the decoding order of the SIC-based scheme has a significant effect on the performance of the GLST system. Similarly to the classic decoding scheme using the optimum decoding method developed in [5], in our group-based optimum ordered decoding scheme, the higher the post-detection SNR of a specific layer, the earlier the layer is chosen to be detected. Without loss of generality, the decoding order in Fig. 3 is assumed to be $\left\{1^{\prime}, 2^{\prime}, \ldots, q^{\prime}\right\}$. We can see that the first iteration is constituted by a STC-group-based SIC operation, i.e. by cancelling the effects of the other $(q-1)$ number of STC-coded layers, also ensuring that temporal interleaving and temporal deinterleaving is carried out according to Fig. 2. At the first decoding layer $1^{\prime}$, the received signal is firstly passed through an interference nulling (IN) module which suppresses all the interfering signals of the other layers using zero-forcing (ZF) and space-time decoded afterwards accordingly. The re-modulated signal multiplied by the corresponding channel $H_{1}$, is then cancelled from the original received signal by the interference cancellation (IC) module. The resultant composite signal of the remaining $(q-1)$ STC-protected layers is then forwarded to the next iteration. This procedure is continued until all $q^{\prime}$ layers are decoded.

In the subsequent iterations, since all groups have been decoded already, the interference nulling is no longer needed, but the interference cancellation is still performed for all STCprotected layers. Each iteration consists of $q$ layers, and each STC-protected layer is processed successively in the same order as in the first iteration. After a number of iterations, full receive diversity of order $N_{r}$ may be achieved for all layers. Compared to the ML detection, this iterative decoding scheme is capable of approaching the same order of receive diversity, despite imposing only a fraction of the computational complexity of ML-style joint detection.

\section{Iterative ML Detection}

The iterative ML detection procedure of the GLST scheme is shown in Fig. 4. Firstly, the $N_{t}$ transmitted $M$-ary symbols 


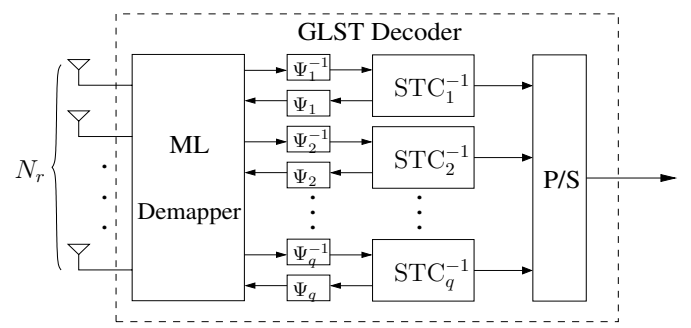

Fig. 4. Iterative ML detection of the GLST scheme, where $\Psi_{j}$ is the corresponding temporal interleaver and $\mathrm{STC}_{j}^{-1}$ represents $j$-layer space-time decoder.

are jointly detected as a combined $\left[N_{t} \times \log _{2} M\right]$-bit symbol by an ML demapper. Then, the probability vector of each $\left[N_{t} \times\right.$ $\log _{2} M$ ]-bit symbol is converted to $q$ number of probability vectors corresponding to the $q$ number of $\left[N_{t}^{j} \times \log _{2} M\right]$ bit symbols. The resultant probability vectors are then fed to the component STTC decoders. Iterative gains can be attained, since the EXIT curve of the ML demapper is a slanted line although this is not explicitly shown here owing to space-economy. However, it is not guaranteed that the DCMC capacity, as detailed later in Section III-D, can be achieved with the aid of iterative ML detection, because the maximum achievable rate of the iterative ML-based scheme is dependent on the EXIT curve shapes of the various space-time trellis codes.

\section{Capacity and Maximum Achievable Rate}

In the context of discrete-amplitude QAM [19] and PSK [19] modulation, we encounter a Discrete-input Continuous-output Memoryless Channel (DCMC) [19]. In order to design a near-capacity coding scheme, we derive the bandwidth efficiency $\eta$ of various SIC-based GLST schemes for transmission over the DCMC based on the properties of EXIT charts [20]. In this contribution, we consider the scenario when $N_{t}=4$ transmit and $N_{r}=4$ receive antennas are used, where $N_{t}^{1}=N_{t}^{2}=2$. For simplicity, the component STCs utilized for all groups are assumed to be identical. Specifically, we use the 4-state based STTC-4 [8, Fig. 4] and 16-state based STTC-16 [8, Fig. 5] as the component STCs of GLST schemes, respectively.

It was argued in [13], [20] that the maximum achievable bandwidth efficiency of the system is equal to the area under the EXIT curve of the inner code, provided that the channel's input is independently and uniformly distributed as well as assuming that the inner code rate is 1 and the MAP decoding algorithm is used. Although these properties were formally proven for the family of Binary Erasure Channels (BECs) [20], they have also been observed to hold for AWGN, Rayleigh and multipath communication channels [13], [20]. Assuming that the area under the EXIT curve of the inner decoder, i.e. the GLST decoder, is represented by $A_{E}$, the maximum achievable rate curves of various SIC and ML based GLST schemes are shown in Fig. 5 together with the DCMC capacity curves of the multiplexing-based MIMO scheme, which employed a ML detector. The capacity curve of the unrestricted Continuous-input Continuous-output Memoryless Channel (CCMC) [4], [19] is also depicted in Fig. 5 for comparison. As shown in Fig. 5,

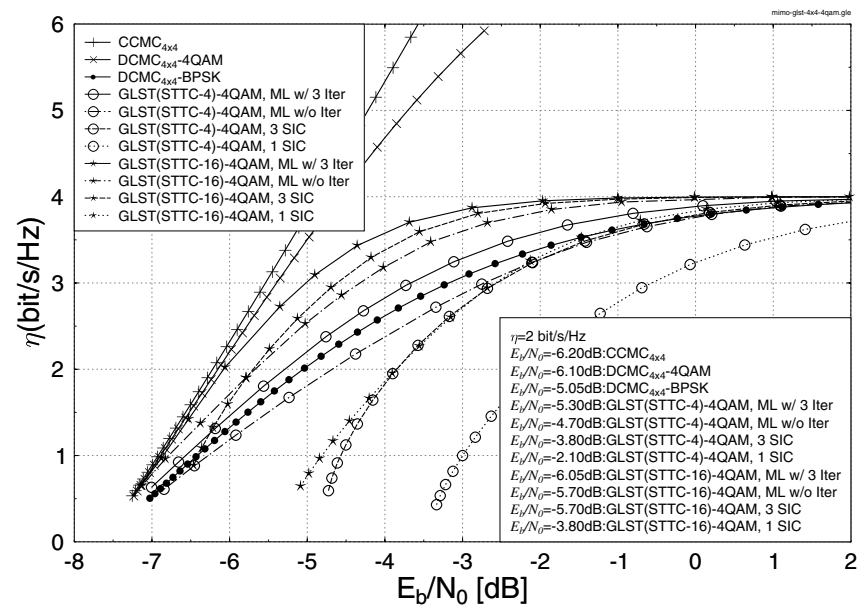

Fig. 5. The capacity and maximum achievable rate of various SIC and ML based GLST schemes, when communicating over uncorrelated flat Rayleigh fading channels employing $N_{t}=4$ transmit antennas and $N_{r}=4$ receive antennas.

the DCMC capacity of the multiplexing-based MIMO scheme employing 4QAM ( DCMC $\left._{4 \times 4}-4 \mathrm{QAM}\right)$ is higher than that of all the GLST schemes and may be regarded as the tight upper capacity bound of the $(4 \times 4)$-element 4 QAM MIMO systems, while the multiplexing-based MIMO scheme using BPSK $\left(\mathrm{DCMC}_{4 \times 4}\right.$-BPSK) exhibits a much lower DCMC capacity. For a specific GLST scheme, the maximum achievable rate improves significantly, when invoking three SIC operations instead of a single one, while any further improvements remain marginal after three SICs, since full receive diversity was approached. As no iterations can be carried out between the SIC-based demodulator and the STTC decoder when using SIC-based detection, some information loss occurs which cannot be recovered. For comparison, the maximum achievable rate of the ML based detection scheme is also given in Fig. 5. The number of iterations between the ML demapper and GLST component space-time decoders was kept to three, which is the same as the number of SIC iterations. Hence, both schemes invoke the same number of space-time decoder operations but the ML demapper exhibits a higher complexity compared to the interference cancellation operation. As shown in Fig. 5, the ML-based scheme provides a higher maximum achievable rate than that of the SIC-based arrangement.

\section{System DESIGN AND EXIT Chart ANALYSIS}

The main objective of employing EXIT charts [11] is to analyse the convergence behaviour of iterative decoders by examining the evolution of the input/output mutual information exchange between the inner and outer decoders during the consecutive iterations. As mentioned in Section III-D, the area under the EXIT curve of the inner decoder is approximately equal to the channel capacity, when the channel's input is independently and uniformly distributed. Similarly, the area under the EXIT curve of the outer code is approximately equal to $(1-R)$, where $R$ is the outer code rate. Furthermore, our experimental results show that an intermediate URC changes only the shape but not the area under the EXIT curve of the inner code. A narrow but marginally open EXIT-tunnel 
in an EXIT chart indicates the possibility of achieving a nearcapacity performance. Therefore, we invoke IRCCs for the sake of appropriately shaping the EXIT curves by minimizing the area in the EXIT-tunnel using the procedure of [12], [13].

An IRCC constituted by a set of $P=17$ subcodes was constructed in [13] from a systematic, rate-1/2, memory-4 mother code defined by the generator polynomial $\left(1, g_{1} / g_{0}\right)$, where $g_{0}=1+D+D^{4}$ is the feedback polynomial and $g_{1}=1+D^{2}+D^{3}+D^{4}$ is the feedforward one. Higher code rates may be obtained by puncturing, while lower rates are created by adding more generators and by puncturing under the constraint of maximizing the achievable free distance. In the proposed system the two additional generators are $g_{2}=1+D+D^{2}+D^{4}$ and $g_{3}=1+D+D^{3}+D^{4}$. The resultant $P=17$ subcodes have coding rate spanning from $0.1,0.15,0.2, \ldots$, to 0.9 .

The EXIT function of an IRCC can be obtained by superimposing those of its subcodes. More specifically, the EXIT function of the target IRCC is the weighted superposition of the EXIT functions of its subcodes [13]. Hence, a careful selection of the weighting coefficients could produce an outer code EXIT curve that matches closely the EXIT curve of the inner code. When the area between the two EXIT curves is minimized, decoding convergence to an infinitesimally low BER would be achieved at the lowest possible SNR.

In this paper, we consider an average coding rate of $R=0.5$ for the IRCC outer code. Hence the effective throughput is $2 \times R \log _{2} 4=2 \mathrm{bit} / \mathrm{s} / \mathrm{Hz}$ when 4QAM modulation is employed by the above-mentioned GLST schemes. The channel capacity and maximum achievable rate computed according to the properties of EXIT charts [13], [20] at a throughput of $\eta=2$ bit/s/Hz are depicted in Fig. 5. The exchange of extrinsic information in the schematic of Fig. 1 is visualised by plotting the EXIT characteristics of the inner amalgamated "URC-GLST" decoder and the outer IRCC decoder in Fig. 6 and 7. Note that for both the GLST(STTC-4) and GLST(STTC-16) schemes, where the EXIT curve of the SIC-based GLST decoder is a slanted line, extrinsic information exchange using decoding iterations between the URC decoder and the GLST decoder is needed in order to achieve a near-capacity performance. When there is no iteration between the URC decoder and the GLST decoder, the EXIT curve shape of the URC decoder depends on the first $I_{E}$ value of the GLST decoder. Hence, both the GLST(STTC-4)-URC and GLST(STTC-16)-URC schemes require a higher $E_{b} / N_{0}$ value in order to maintain an area of $A_{E}=0.5$, as shown in Fig. 6 and 7, respectively. In other words, information loss will occur if there is no iteration between the URC decoder and the GLST decoder.

\section{Simulation Results And Discussions}

As we can see from Fig. 6 and 7, the Monte-Carlo simulation based decoding trajectory of the GLST-URC-IRCC schemes using SIC detection within the GLST decoder have a mismatch with their EXIT curves. This is due to the correlated non-zero error propagation between different layers in the process of SIC operation. We found that the higher the correlation of the STTC coded bits, the higher the mismatch.

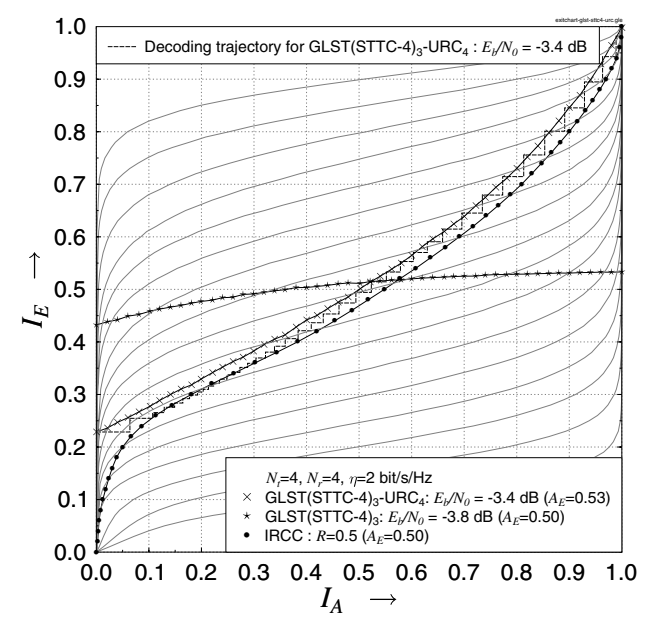

Fig. 6. The EXIT chart curves for the GLST(STTC-4)-URC, IRCC and the IRCC subcodes, when communicating over uncorrelated flat Rayleigh fading channels using $N_{t}=4$ and $N_{r}=4$. The notation GLST(STTC-4) 3 indicates 3 SIC iterations in GLST decoder, and the subscript of URC denotes the number of iterations between the GLST(STTC-4) and URC decoders.

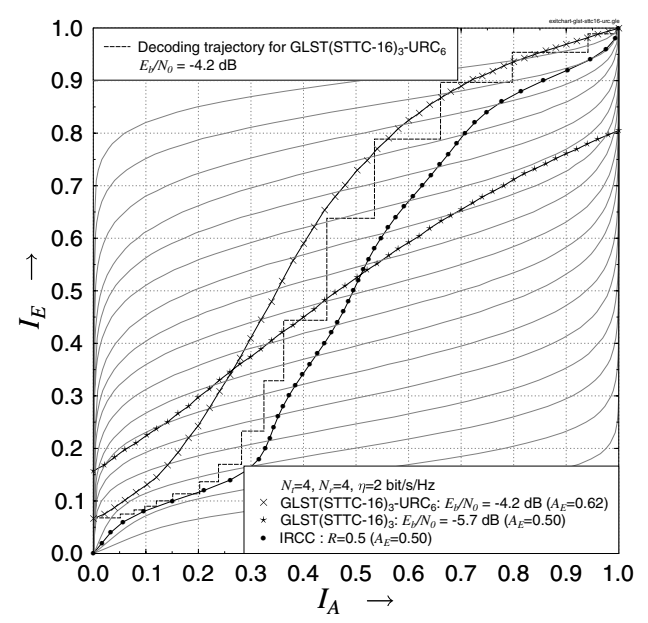

Fig. 7. The EXIT chart curves for the GLST(STTC-16)-URC, IRCC and the IRCC subcodes, when communicating over uncorrelated flat Rayleigh fading channels using $N_{t}=4$ and $N_{r}=4$. The notation GLST(STTC-16) 3 indicates 3 SIC iterations in GLST decoder, and the subscript of URC denotes the number of iterations between the GLST(STTC-16) and URC decoders.

As the number of trellis states increases from 4 to 16 , the correlation of the STTC-16 coded bits becomes higher, because the code imposes more correlation. As a result, the STTC-16 scheme will yield a higher correlation than that of the STTC-4 scheme, when there is error propagation between the different layers due to the imperfect SIC operation. Hence, the decoding trajectory of the GLST(STTC-16)-URCIRCC scheme has a higher mismatch in its EXIT curve. For comparison, the decoding trajectory of the GLST(STTC-16)URC-IRCC scheme employing ML detection is also presented in Fig. 8. Again, we use three iterations between the ML demapper and GLST component decoders so that the number of STTC decoder operations invoked is the same as that of the scheme employing three SIC iterations. Since there is no error propagation in the ML-based scheme, the decoding trajectory of the ML-based scheme matches its EXIT curve well. However, the error propagation was avoided in the ML- 


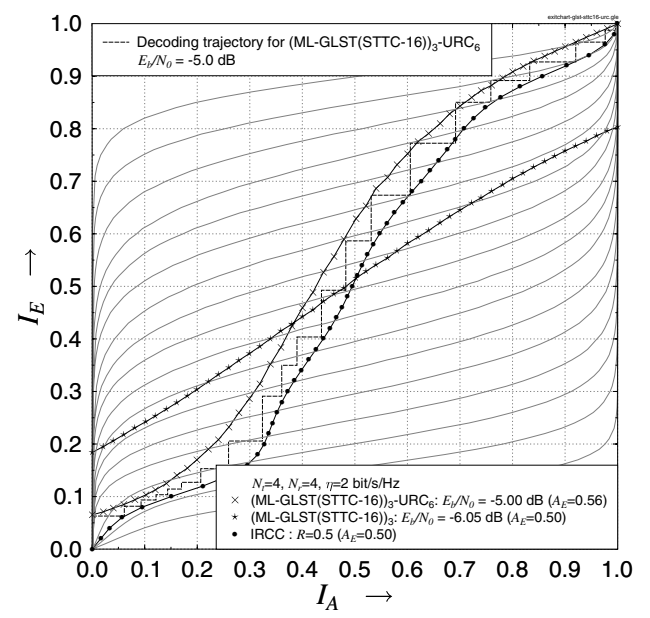

Fig. 8. The EXIT chart curves for the ML-GLST(STTC-16)-URC, IRCC and the IRCC subcodes, when communicating over uncorrelated flat Rayleigh fading channels using $N_{t}=4$ and $N_{r}=4$. The notation (ML-GLST(STTC-16) $)_{3}$ indicates 3 iterations between ML demapper and GLST component decoders, and the subscript of URC denotes the number of iterations between the ML-GLST(STTC-16) and URC decoders.

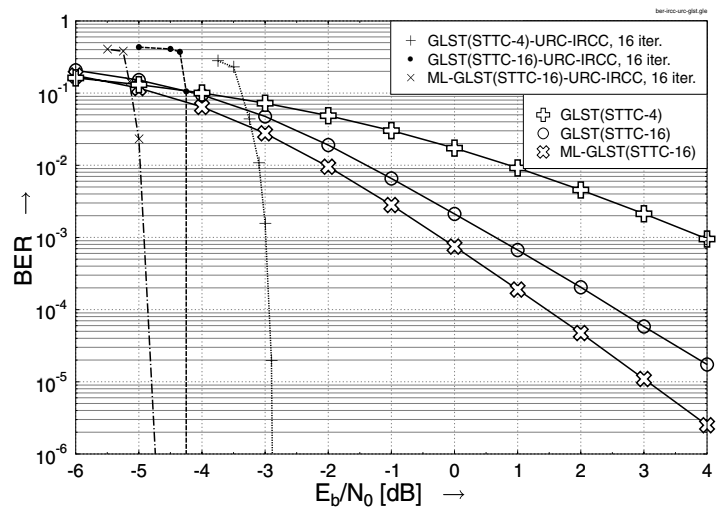

Fig. 9. The BER performance comparison of the GLST-URC-IRCC and of the stand-alone GLST schemes with iterative SIC and ML based GLST decoder.

based scheme at the price of a higher complexity.

Despite the mismatch observed in the EXIT charts, we were able to design near-capacity SIC schemes. Fig. 9 presents the BER performance of the GLST-URC-IRCC scheme employing various space-time trellis codes combined with SIC detection. It is clearly shown that the GLST(STTC-4)-URCIRCC scheme is capable of performing within 0.4-0.5 dB of the corresponding maximum achievable rate obtained from the EXIT charts. On the other hand, the GLST(STTC-16)URC-IRCC scheme, where a high mismatch of the decoding trajectory to its EXIT curve occurs, performs closer to the $\mathrm{DCMC}_{4 \times 4}$-4QAM capacity. The BER performance of the most complex ML-detected GLST(STTC-16)-URC-IRCC scheme is also depicted in Fig. 9, where we can see a 0.7 $\mathrm{dB}$ gain over the SIC based scheme. However, the complexity of the ML-based scheme is unaffordable, especially when the number of GLST layers is high.

\section{Conclusions}

In this contribution, we have proposed a low-complexity SIC-based iteratively decoded GLST-URC-IRCC scheme de- signed with the aid of EXIT chart analysis. We quantified the maximum achievable rates of the GLST schemes using various STTCs. According to the simulation results, we found that the iterative GLST(STTC)-URC-IRCC scheme using SIC detection strikes an attractive trade-off between the complexity imposed and the effective throughput achieved.

\section{REFERENCES}

[1] G. J. Foschini and M. J. Gans, "On limits of wireless communications in a fading environment when using multiple antennas," Wireless Personal Communications, vol. 6, pp. 311-335, March 1998.

[2] I. E. Telatar, "Capacity of multi-antenna Gaussian Channels," European Transactions on Telecommunication, vol. 10, pp. 585-595, Nov./Dec. 1999.

[3] M. S. Alouini and A. J. Goldsmith, "Capacity of Rayleigh fading channels under different adaptive transmission and diversity-combining techniques," IEEE Transactions on Vehicular Technology, vol. 48, pp. 1165-1181, July 1999.

[4] S. X. Ng and L. Hanzo, "On the MIMO channel capacity of multidimensional signal sets," IEEE Transactions on Vehicular Technology, vol. 55, pp. 528-536, March 2006.

[5] P. W. Wolniansky and G. J. Foschini and G. D. Golden and R. A. Valenzuela, "V-BLAST: an architecture for realizing very high data rates over the rich-scattering wireless channel," in 1998 URSI International Symposium on Signals, Systems, and Electronics, (Pisa, Italy), pp. 295300, 1998.

[6] S. M. Alamouti, "A simple transmit diversity technique for wireless communications," IEEE Journal on Selected Areas in Communications, vol. 16, pp. 1451-1458, Oct. 1998.

[7] V. Tarokh, H. Jafarkhani, and A. R. Calderbank, "Space-time block codes from orthogonal designs," IEEE Transactions on Information Theory, vol. 45, pp. 1456-1467, July 1999.

[8] V. Tarokh, N. Seshadri, and A. R. Calderbank, "Space-time codes for high data rate wireless communication: Performance criterion and code construction," IEEE Transactions on Information Theory, vol. 45, pp. 744-765, Mar. 1998.

[9] V. Tarokh, A. Naguib, N. Seshadri, and A. R. Calderbank, "Combined array processing and space-time coding," IEEE Transactions on Information Theory, vol. 45, pp. 1121-1128, May 1999.

[10] M. Tao and R. S. Cheng, "Generalized layered space-time codes for high data rate wireless communications," IEEE Transactions on Wireless Communications, vol. 3, pp. 1067-1075, July 2004.

[11] S. ten Brink, "Convergence behaviour of iteratively decoded parallel concatenated codes," IEEE Transactions on Communications, vol. 49, pp. 1727-1737, Oct. 2001.

[12] M. Tüchler and J. Hagenauer, "EXIT charts of irregular codes," in Proceeding of the 36th Annual Conference on Information Sciences and Systems [CDROM], (Princeton, NJ, USA), March 2002.

[13] M. Tüchler, "Design of serially concatenated systems depending on the block length," IEEE Transactions on Communications, vol. 52, pp. 209218, Feb. 2004.

[14] M. Tüchler, "Convergence prediction for iterative decoding of threefold concatenated systems," in GLOBECOM'02, vol. 2, (Taipei, Taiwan), pp. 1358-1362, 17-21 November 2002.

[15] S. X. Ng, J. Wang, M. Tao, L.-L. Yang, and L. Hanzo, "Iteratively decoded variable-length space-time coded modulation: code construction and convergence analysis," IEEE Transactions on Wireless Communications, vol. 6, pp. 1953-1963, May 2007.

[16] L. Hanzo, T. H. Liew and B. L. Yeap, Turbo Coding, Turbo Equalisation and Space Time Coding for Transmission over Wireless channels. New York, USA: John Wiley IEEE Press, 2002.

[17] G. D. Golden, C. J. Foschini, R. A. Valenzuela, and P. W. Wolniansky, "Detection algorithm and initial laboratory results using V-BLAST space-time communication architecture," Electronics Letters, vol. 35, pp. 14-16, Jan. 1999.

[18] G. J. Foschini, "Layered space-time architecture for wireless communication in a fading environment when using multiple antennas," Bell Labs Technical Journal, vol. 1, pp. 41-59, Autumn 1996.

[19] J. G. Proakis, Digital Communications. 4th ed. New York: McGraw-Hill, 2001.

[20] A. Ashikhmin, G. Kramer, and S. ten Brink, "Extrinsic information transfer functions: model and erasure channel properties," IEEE Transactions on Information Theory, vol. 50, pp. 2657-2673, Nov. 2004. 